\title{
Charge and Spin Transport in a Metal-Semiconductor Heterostructure with Double Schottky Barriers
}

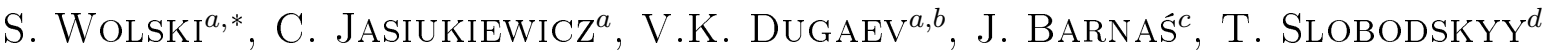 \\ AND W. HANSEN ${ }^{d}$ \\ ${ }^{a}$ Department of Physics, Rzeszów University of Technology, Al. Powstańców Warszawy 6, 35-959 Rzeszów, Poland \\ ${ }^{b}$ Departamento de Física and CeFEMA, Instituto Superior Técnico, Universidade de Lisboa, \\ Av. Rovisco Pais, 1049-001 Lisbon, Portugal \\ ${ }^{c}$ Faculty of Physics, Adam Mickiewicz University, ul. Umultowska 85, 61-614 Poznań, Poland \\ ${ }^{c}$ Institute for Applied Physics, University of Hamburg, \\ ${ }^{d}$ Jungiusstraße 11, 20355 Hamburg, Germany
}

\begin{abstract}
Taking into account the available experimental results, we model the electronic properties and current-voltage characteristics of a ferromagnet-semiconductor junction. The Fe $/ \mathrm{GaAs}$ interface is considered as a Fe $/(i-\mathrm{GaAs}) / n^{+}-$ GaAs/n-GaAs multilayer structure with the Schottky barrier. We also calculate numerically the current-voltage characteristics of a double-Schottky-barrier structure Fe/GaAs/Fe, which are in agreement with available experimental data. For this structure, we have estimated the spin current in the GaAs layer, which characterizes spin injection from the ferromagnet to the semiconductor.
\end{abstract}

DOI: 10.12693/APhysPolA.127.472

PACS: $73.30 .+\mathrm{y}, 73.40 .-\mathrm{c}, 75.76 .+\mathrm{j}$

\section{Introduction}

One of the key issues in spintronics is the problem of efficient spin injection and spin manipulation in semiconductors [1]. It is well known that when spin-polarized electrons are directly transmitted from a ferromagnetic metal into a semiconductor, efficiency of such spin injection is usually very low $[2,3]$ because of a large difference in the conductivities of the metal and semiconductor $[4,5]$. However, a tunneling barrier between the metal and semiconductor can help in maintaining the spin efficiency relatively high $[6,7]$. Accordingly, the Schottky barrier at the metal-semiconductor interface [8] attracted a lot of attention since it can act as a natural tunnel barrier separating the semiconductor and the ferromagnetic metal.

The Schottky barrier [9-10] with optimal parameters for spin injection can appear only at certain conditions related to the physical processes at the metalsemiconductor heterojunction. All the parameters of the Schottky barrier, such as its height and width and the energy profile, mostly depend on the distribution of dopants in the semiconductor near the interface. When the external voltage is applied to the barrier, the accumulated electric charge related to redistribution of electrons and holes strongly affects the energy profile. Apart from this, a nonequilibrium spin accumulation appears near the ferromagnet-semiconductor interface, which affects

\footnotetext{
* corresponding author; e-mail: wolski@prz.edu.pl
}

transport of electrons and holes in different spin channels.

Our main objective is to find optimal regimes of charge and spin transport through the Schottky barrier, where spin injection to the semiconductor is efficient. To do this we simulate physical processes in the junction, taking into account modification of the electronic energy structure near the interface as well as the spin and charge accumulation in a nonequilibrium situation. As a result of the numerical simulations, we obtained the currentvoltage characteristics of the $\mathrm{Fe} / \mathrm{GaAs} / \mathrm{Fe}$ junction with a double Schottky barrier. These results are in satisfactory agreement with the $I-V$ characteristics obtained experimentally on the $\mathrm{Fe} / n^{+}-\mathrm{GaAs} / n$-GaAs $/ n^{+}-\mathrm{GaAs} / \mathrm{Fe}$ heterostructure which works as the double Schottky barrier.

\section{Model}

We describe the charge and spin transport in ferromagnet-semiconductor structures in terms of a semiclassical model, with thermally activated electrons and holes near the metal-semiconductor interface. This model properly describes transport properties at room temperatures, where the thermally activated conductivity is much larger than the under-barrier tunneling. In the semiclassical approximation we have to calculate spin-resolved profiles for the electrostatic potential, spinpolarized electron and hole densities, and the chemical potential. The key point of the simulations is selfconsistency, which is especially important for a large deviation of the system from equilibrium. The heterostructure under consideration is shown schematically in Fig. 1. 


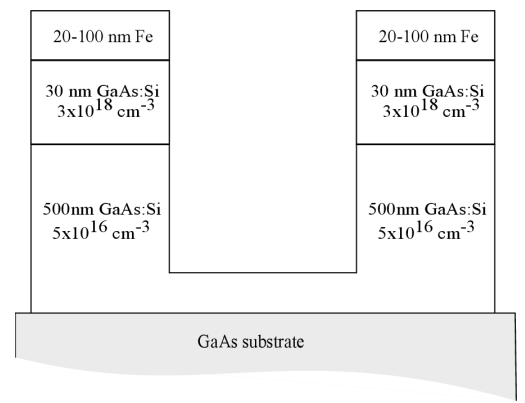

Fig. 1. Schematic view of the $\mathrm{Fe} / \mathrm{GaAs} / \mathrm{Fe}$ heterostructure with two Schottky junctions.

The doping concentration is $N_{D}=5 \times 10^{16} \mathrm{~cm}^{-3}$. For lower doping concentration, the conductance is significantly reduced. The $30 \mathrm{~nm} n^{+}$-GaAs layer of enhanced donor concentration assures efficient spin injection trough the barrier $[11,12]$.

The Poisson equation, which accounts for space distribution of the total charge, determines the electrostatic potential $\phi(x)$,

$$
-\nabla^{2} \phi=4 \pi e\left(n+N_{a}^{-}-N_{d}^{+}-p\right),
$$

where the electron and hole concentrations, $n=n(x)=$ $n_{\uparrow}(x)+n_{\downarrow}(x)$ and $p=p(x)=p_{\uparrow}(x)+p_{\downarrow}(x)$, respectively, are calculated from the corresponding spin components determined by spin-resolved chemical potentials $\mu_{\sigma}(x)$, while $N_{a}^{-}(x)$ and $N_{d}^{+}(x)$ are the densities of ionized acceptors and donors. The electric current in each spin channel is related to the gradient of the corresponding chemical potential, $j_{n \sigma}=\mu_{n} n_{\sigma} \nabla \mu_{\sigma}$ for electrons and $j_{p \sigma}=\mu_{p} p_{\sigma} \nabla \mu_{\sigma}$ for holes, where $\mu_{n}$ and $\mu_{p}$ are the mobilities of electrons and holes. The difference of chemical potentials in each spin channel at the ends of a sample is determined by the external voltage $V$.

The energy band diagram in Fig. 2 shows schematically the equilibrium profiles of the conductance $\left(\varepsilon_{c}\right)$ and valence $\left(\varepsilon_{v}\right)$ band edges in GaAs in the double junction structure. The curvature of the band edges is due to the spatial variation of electrostatic potential, $\varepsilon_{c, v}=\varepsilon_{c 0, v 0}+e \phi$, where $\varepsilon_{c 0, v 0}$ describe the band edges in the corrresonding bulk semiconductor.

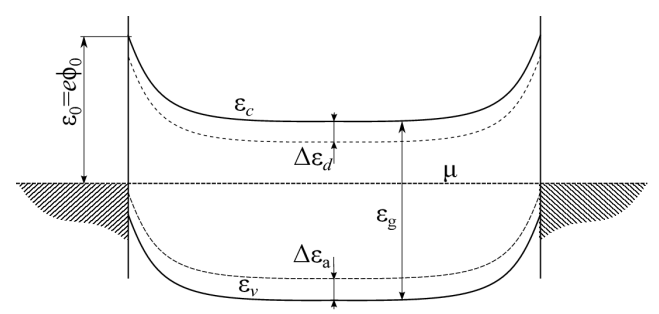

Fig. 2. Energy band diagram of the double metalsemiconductor junction in thermal equilibrium. Here $\mu$ is the equilibrium chemical potential, $\Delta \varepsilon_{d}$ and $\Delta \varepsilon_{a}$ describe the donor and acceptor levels, respectively, $\varepsilon_{g}$ is the energy gap, while $\varepsilon_{0}$ denotes the work function.

\section{Results}

The results of numerical calculations of the distribution of electrons, holes and ionized donors in the biased $\mathrm{Fe} / n^{+}-\mathrm{GaAs} / n$-GaAs $/ n^{+}-\mathrm{GaAs} / \mathrm{Fe}$ heterostructure are presented in Fig. 3. Here we take $N_{D}=5 \times 10^{16} \mathrm{~cm}^{-3}$ for $\mathrm{n}$-GaAs, $N_{D}^{+}=3 \times 10^{18} \mathrm{~cm}^{-3}$ for $n^{+}$-GaAs, and $\varepsilon_{0}=0.84 \mathrm{eV}$ for the work function. A constant current $j=5 \times 10^{-6} \mathrm{~A} / \mathrm{cm}^{2}$ flows through the structure due to an external voltage, see also the inset of Fig. 3. One can also note, that variation of the electron density is correlated with the density of ionized donors $N_{d}^{+}(x)$ within the entire sample. From detailed analysis of the technology process and available experimental data on $\mathrm{Fe} / \mathrm{GaAs} / \mathrm{Fe}$ structures [13] follows that an important element of the modeling of electric properties of such structures is an additional thin layer of $i$-GaAs (GaAs strongly doped with $\mathrm{Fe}$ ), which can be formed in the vicinity of $\mathrm{Fe} / \mathrm{GaAs}$ interface $[14,15]$. This is due to diffusion of Fe atoms into GaAs, which strongly affect the properties of GaAs semiconductor making it almost insulating due to the deepacceptor properties of $\mathrm{Fe}$ in GaAs [16]. The variation of $I-V$ characteristics with the change of acceptor density in the $n^{+}$-GaAs layer is presented in Fig. 4.

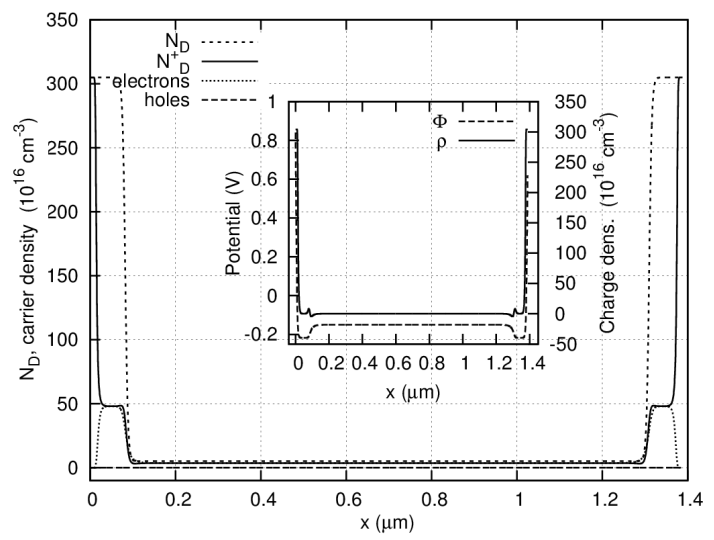

Fig. 3. Spatial distribution of donors, electrons and holes in $\mathrm{Fe} / n^{+}-\mathrm{GaAs} / n$-GaAs $/ n^{+}$-GaAs/Fe heterostructure under a constant current. The inset shows the profiles of the electrostatic potential and the charge density along the sample.

Due to the ferromagnetic elements of the junction, a spin accumulation may appear in the nonequilibrium state. This accumulation can be included in the numerical simulations in terms of the approach developed in Refs. [17] and [4]. In this approach, the spatial variation of the chemical potential is described by the following equation

$$
\frac{\mathrm{d} \mu_{\uparrow, \downarrow}}{\mathrm{d} x}=-\frac{e}{\sigma_{\uparrow, \downarrow}} j_{\uparrow, \downarrow},
$$

with linear relations between the spin-resolved and total conductivities and currents

$$
\sigma_{\uparrow}=\alpha \sigma, \quad \sigma_{\downarrow}=(1-\alpha) \sigma,
$$




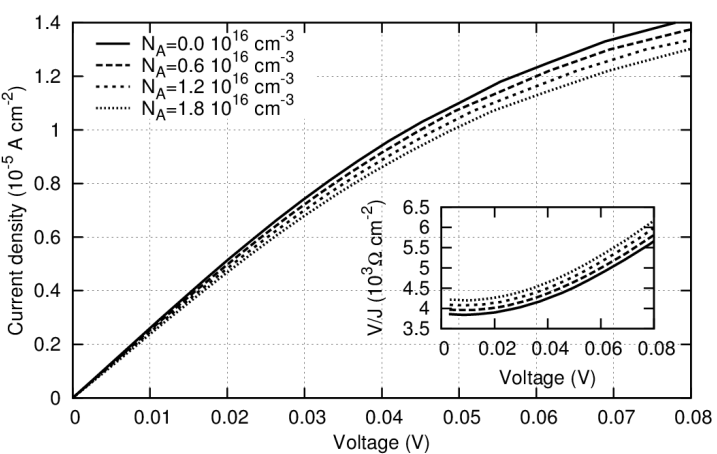

Fig. 4. Voltage dependence of electric current for different acceptor density in $n^{+}$-GaAs layer. The inset shows the dependence of resistivity on applied voltage for different densities of acceptors.

$$
j_{\uparrow}=\beta j, \quad j_{\downarrow}=(1-\beta) j,
$$

where the coefficients $\alpha$ and $\beta$ describe specific properties of the junction. Taking into account Eqs. (2) and (3), one arrives at the following equation for the difference between electrochemical potentials for the spin-up and spin-down electron channels,

$$
\frac{\mathrm{d}\left(\mu_{\uparrow}-\mu_{\downarrow}\right)}{\mathrm{d} x}=-\frac{\beta-\alpha}{\alpha(\alpha-1)} \frac{e j}{\sigma} .
$$

Having found the electrochemical potential and current in both spin channels, one can calculate the spin current density $j_{s}$ as $j_{s}=\hbar\left(j_{\uparrow}-j_{\downarrow}\right) / 2 e$.

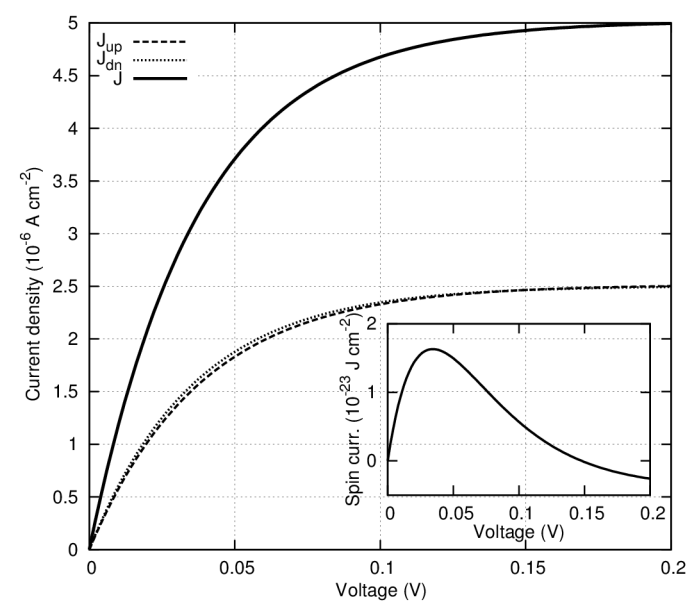

Fig. 5. Current-voltage and spin-current-voltage characteristics for $\alpha=0.501$ and $\beta=0.5$.

The results of numerical calculation of the charge and spin current densities are presented in Fig. 5. For simplicity, we assumed there some effective values of the parameters $\alpha$ and $\beta ; \alpha=0.501$ and $\beta=0.5$. We note that the spin polarization of transferred current strongly depends on the factors $\alpha$ and $\beta$, which characterize the interfaces. In particular, at the ferromagnetic side of the interface, they are determined mainly by the diffusion constant and spin-flip relaxation time [17].

\section{Conclusions}

Using the semiclassical approach we have calculated the current-voltage characteristics and spin current in $\mathrm{Fe} / \mathrm{GaAs} / \mathrm{Fe}$ double-Schottky-barrier structures. Our results are in satisfactory agreement with experimental results of Ref. [13]. We have found that the electrical properties of $\mathrm{Fe} / \mathrm{GaAs}$ strongly depend on the formation of an additional thin insulating $i$-GaAs layer near the interface due to diffusion of Fe atoms into GaAs. The spin injection through the interface is mostly determined by the conductive properties of Fe near the interface and by the spin-flip scattering in this region.

\section{Acknowledgments}

This work is supported by the National Center of Research and Development in Poland in frame of EU project Era.Net.Rus "SpinBarrier".

\section{References}

[1] I. Žutic, J. Fabian, S. Das Sarma, Rev. Mod. Phys. 76, 323 (2004).

[2] W.Y. Lee, S. Garedelis, B.C. Choi, Y.B. Xu, C.G. Smith, C.H. Barnes, A.D. Ritchi, E.H. Linfield, J.A.C. Bland, J. Appl. Phys. 85, 6682 (1999).

[3] P.R. Hammar, B.R. Bennet, M.J. Yang, M. Johnson, Phys. Rev. Lett. 83, 203 (1999).

[4] G. Schmidt, D. Ferrand, L.W. Molenkamp, A.T. Filip, B.J. van Wees, Phys. Rev. B. 62, R4790 (2000).

[5] A. Khaetskii, J.C. Egues, D. Loss, C. Gould, G. Schmidt, L.W. Molenkamp, Phys. Rev. B 71, 235327 (2005).

[6] E.I. Rashba, Phys. Rev. B 62, R16267 (2000).

[7] D.L. Smith, R.N. Silver, Phys. Rev. B 64, 045323 (2001).

[8] S.M. Sze, K.K. Ng, Physics of Semiconductor Devices, Wiley, Hoboken 2007.

[9] E.H. Rhoderick, Solid-State, Electron Devices, IEE Proceedings I 129, 1 (1982).

[10] R.T. Tung, Appl. Phys. Rev. 1, 011304 (2014).

[11] Q. O. Hu, E.S. Garlid, P.A. Crowell, C.J. Palmstrøm, Phys. Rev. B 84, 085306 (2011).

[12] S.A. Crooker, E.S. Garlid, A.N. Chantis, D.L. Smith, K.S.M. Reddy, Q.O. Hu, T. Kondo, C.J. Palmstrøm, P.A. Crowell, Phys. Rev. B 80, 041305(R) (2009).

[13] T. Nickiel, Structural, electrical, magnetic properties of iron layers grown on (001)GaAs, Thesis, Universität Hamburg, 2012.

[14] D.T. McInturff, E.S. Harmon, J.P.C. Chang, T.M. Pekarek, J.M. Woodall, Appl. Phys. Lett. 69, 1885 (1996).

[15] P. Schieffer, A. Guivarc'h, C. Lallaizon, B. Lépine, D. Sébilleau, P. Turban, C. Jézéquel, Appl. Phys. Lett. 89, 161923 (2006).

[16] M. Kleverman, P. Omling, L.A. Ledebo, H.G. Grimmeiss, J. Appl. Phys. 54, 814 (1983).

[17] P.C. van Son, H. van Kempen, P. Wyder, Phys. Rev. Lett. 58, 2271 (1987). 